\title{
Colonic polyps: inheritance, susceptibility, risk evaluation, and diagnostic management
}

This article was published in the following Dove Press journal:

Cancer Management and Research

29 December 2010

Number of times this article has been viewed

\section{Emanuele Neri \\ Lorenzo Faggioni \\ Lorenzo Cini \\ Carlo Bartolozzi}

Diagnostic and Interventional Radiology, University of Pisa, Pisa, Italy
Correspondence: Emanuele Neri

Diagnostic and Interventional Radiology,

University of Pisa,Via Paradisa, 2-56100

Pisa, Italy

Tel/fax +39 0509973/3

Email neri@med.unipi.it
Abstract: Colorectal cancer (CRC) is the third-ranked neoplasm in order of incidence and mortality, worldwide, and the second cause of cancer death in industrialized countries. One of the most important environmental risk factors for CRC is a Western-type diet, which is characterized by a low-fiber and high-fat content. Up to $25 \%$ of patients with CRC have a family history for CRC, and a fraction of these patients are affected by hereditary syndromes, such as familial adenomatous polyposis, Gardner or Turcot syndromes, or hereditary nonpolyposis colorectal cancer. The onset of CRC is triggered by a well-defined combination of genetic alterations, which form the bases of the adenoma-carcinoma sequence hypothesis and justify the set-up of CRC screening techniques. Several screening and diagnostic tests for CRC are illustrated, including rectosigmoidoscopy, optical colonoscopy (OC), double contrast barium enema (DCBE), and computed tomography colonography (CTC). The strengths and weaknesses of each technique are discussed. Particular attention is paid to CTC, which has evolved from an experimental technique to an accurate and mature diagnostic approach, and gained wide acceptance and clinical validation for CRC screening. This success of CTC is due mainly to its ability to provide cross-sectional analytical images of the entire colon and secondarily detect extracolonic findings, with minimal invasiveness and lower cost than OC, and with greater detail and diagnostic accuracy than DCBE. Moreover, especially with the advent and widespread availability of modern multidetector CT scanners, excellent quality 2D and 3D reconstructions of the large bowel can be obtained routinely with a relatively low radiation dose. Computer-aided detection systems have also been developed to assist radiologists in reading CTC examinations, improving overall diagnostic accuracy and potentially speeding up the clinical workflow of CTC image interpretation.

Keywords: colonic polyps, colorectal cancer, colonoscopy, double contrast barium enema, computed tomography colonography

\section{Colorectal cancer: epidemiology and risk factors}

Colorectal cancer (CRC) is the third-ranked neoplasm in order of incidence and mortality worldwide. ${ }^{1}$ It is more frequent in industrialized countries, where it is the second cause of cancer death and is a social and health care issue of major importance. . $^{1,2}$

In 2009, 146,970 new cases of CRC and 49,920 CRC-related deaths occurred in the United States. ${ }^{3,4}$ In the European Union, approximately 220,000 new CRC cases per year are estimated to be diagnosed annually. ${ }^{5}$

The likelihood of developing CRC increases from the second to the ninth decade. $\mathrm{CRC}$ is rare before the age of 40 years, and most cases are diagnosed after the age of 50 . The incidence of CRC is slightly higher in males than in females, especially for rectal 
carcinoma. In developing countries CRC has a lower, yet growing incidence. ${ }^{1}$ Although the etiology of CRC is still unknown, epidemiological and biomolecular studies have unveiled environmental and genetic risk factors that favor the onset of CRC. ${ }^{3}$

One of the most important environmental risk factors for CRC is a Western-type diet, which is characterized by a low fiber and high fat content. CRC-related mortality is directly correlated with per-person calorie intake, high consumption of meat proteins and fat, and high blood cholesterol levels. Conversely, in African populations, in which consumption of fruit and vegetables is higher than in Western countries, the incidence of CRC is much lower. Moreover, it has been observed that people who have migrated to industrialized countries tend to acquire the same $\mathrm{CRC}$ incidence rates of those countries, supporting the hypothesis that geographic differences in CRC incidence are due to dietary habits rather than different genetic patterns. ${ }^{6}$

Research has shown that up to $25 \%$ of patients with CRC have inherited mutations that increase their risk for CRC. A fraction of these patients are affected by well-characterized hereditary syndromes, which can be classified as polyposis and nonpolyposis CRC:

- Familial adenomatous polyposis (FAP; also known as familiar polyposis of the colon) is a rare autosomal dominant condition that causes the growth of hundreds or thousands of adenomatous polyps in the large bowel. It is associated with a deletion in the long arm of chromosome 5, which contains the $A P C$ oncosuppressor gene. CRC develops in almost all affected patients under 40 years. There is a classic form (in which patients typically harbor between 500 and 2500 colonic adenomas) and an attenuated form of FAP, characterized by a lower number of polyps (typically 30 ) that are predominantly located in the proximal colon. Affected patients may also have associated extracolonic diseases, such as desmoid tumors and gastroduodenal carcinomas. ${ }^{7}$

- Gardner syndrome is characterized by a combination of intestinal polyposis (identical to classic FAP) and numerous osteomas, epidermal cysts, fibromatosis, as well as thyroid and duodenal carcinomas and dental abnormalities.

- Turcot syndrome is a rare condition characterized by the association of FAP and tumors of the central nervous system (such as medulloblastoma and glyoblastoma). ${ }^{3}$

- Hereditary nonpolyposis colorectal cancer (HNPCC, also known as Lynch syndrome) is an autosomal dominant hereditary syndrome, associated with germline mutations in genes that result from DNA repair mechanisms (in particular, $M S H 2, M L H 1, P M S$, and $P M S 2$ ), causing microsatellite DNA instability and impaired mismatch repair, and leading to higher risk of cancer development. A strong association exists between HNPCC and endometrial and ovarian carcinomas. ${ }^{8}$ In HNPCC patients, CRC typically occurs earlier than 50 years, that is, 10 to 15 years before the average onset of $\mathrm{CRC}$ in the overall population. Moreover, CRC in HNPCC patients is more frequently located in the cecum or ascending colon. ${ }^{3,9}$

- Chronic inflammatory bowel diseases: CRC is more frequent in patients with ulcerative colitis, in whom the absolute risk of cancer increases at a rate of $0.5 \%$ to $1 \%$ per year after 10 years of disease, with cancer development typically in $8 \%$ to $30 \%$ of cases after 25 years from disease onset. Conversely, in patients with Crohn disease, CRC risk is not significantly higher than in the normal population. ${ }^{3}$

Other conditions that increase the risk for CRC development are:

- Bacteremia related to Streptococcus bovis: for unknown reasons, individuals with a history of $S$. bovis-induced endocarditis or sepsis have a higher incidence of CRC. ${ }^{3}$

- Tobacco smoke, which is associated with the development of colorectal adenomas: several studies have investigated the potential procarcinogenetic role of tobacco smoke, and a dose-response relationship with $\mathrm{CRC}$ has been found for cigarette pack-years, smoking duration, smoking intensity, smoking history in the distant past, and younger age at initiation of smoking. ${ }^{10,11}$

- Alcohol abuse: epidemiological studies have demonstrated that another risk for CRC could arise from the malnutrition caused by lower folate concentrations in heavy alcohol drinkers. However, recent studies have yielded inconsistent findings concerning such a relationship. ${ }^{12-14}$

Despite multiple risk factors increasing the likelihood of CRC development, the majority of individuals (about 75\%) who develop CRC do not have specific risk factors. ${ }^{15}$ Therefore, the population should be stratified into averagerisk individuals (age greater than 50 years, no personal risk factors, no familial history of CRC) and those with moderate (first-degree relative with a history of adenoma or carcinoma, or personal history of large adenoma or carcinoma) and high CRC risk (inflammatory bowel disease or a family history of an inherited CRC syndrome). ${ }^{16,17}$

\section{The adenoma-carcinoma sequence}

The onset of CRC is triggered by a well-defined sequence of genetic alterations that form the etiological basis of the 
adenoma-carcinoma sequence hypothesis and justify the rationale for CRC screening. The validity of this hypothesis is supported by several findings: ${ }^{18}$

- Populations with a high incidence of adenomas have a high prevalence of CRC, and vice versa.

- There is a substantial overlap between the topographic distribution of colorectal adenomas and that of CRC.

- The incidence peak of adenomatous polyps precedes that of CRC by some years.

- The risk of CRC is directly proportional to the number of adenomas, and the substantial certainty of developing $\mathrm{CRC}$ in patients with FAP is an extreme case of this rule.

- Follow-up programs to detect adenomas with resection of suspected lesions are associated with a reduced incidence of CRC.

Polyps with the highest risk of neoplastic degeneration are those characterized by a large size ( $\geq 20 \mathrm{~mm})$, villous histology, and sessile morphology. The time of progression from adenomatous polyp to carcinoma is about 10 years.

More than $90 \%$ of CRCs arise from adenomatous polyps, ${ }^{19}$ while a small fraction of CRCs develop without evidence of an adenomatous precursor, suggesting that some lesions may undergo malignant transformation without passing through a polypoid intermediate phase. Evidence exists that polypectomy can reduce $\mathrm{CRC}$ incidence and mortality by interrupting the adenoma-carcinoma sequence in the colonic segment where the polyp has been excised. ${ }^{20}$

From a genetic point of view, carcinogenesis is characterized by the accumulation of multiple, consecutive mutations in oncogenes and oncosuppressor genes, starting the adenomacarcinoma sequence at the level of the colonic mucosa. The genes most often involved (and the most widely known) are $A P C, k-R A S, p 53, S M A D 2$, and SMAD4. Moreover, mutations involved in DNA mismatch repair may account for the development of $10 \%$ to $15 \%$ of sporadic CRC and HNPCC..$^{18}$

\section{CRC screening}

The knowledge of the biological mechanisms underlying CRC carcinogenesis and their slow progression over time make this neoplasm ideal for the development of screening programs. Furthermore, CRC is a common disease with a high mortality rate, for which risk conditions can be detected and treatment is more effective in early than in advanced disease stages.

To be effective, screening needs to be performed on a large population, and be sensitive, specific, and well tolerated by patients. Furthermore, it is essential that effective treatment is available once diagnosis has been made. Finally, screening must be cost-effective.

Based on the guidelines of the American Cancer Society, techniques currently available for the secondary prevention of CRC (after the age of 50 years) can be classified as follows: ${ }^{21}$

Tests for detecting polyps and CRC:

Flexible rectosigmoidoscopy (every 5 years);

Optical colonoscopy (OC, every 10 years);

Double contrast barium enema (DCBE, every 5 years);

Computerized tomography colonography (CTC, also

known as virtual colonoscopy: every 5 years).

Tests that can make CRC diagnosis:

Fecal occult blood test (FOBT, every year);

Fecal immunochemical tests (every year);

Fecal DNA test (uncertain time interval).

It must be pointed out that individuals with an increased/ high CRC risk should undergo screening with a higher frequency based on their individual risk level. In Western countries, the most common and readily available tests for CRC screening are rectosigmoidoscopy, OC, and FOBT. Since the introduction of FOBT as a CRC screening test in the 1990s, CRC mortality has been reduced; however, the main limitations of FOBT are a low specificity and a low sensitivity in polyp detection (about $10 \%$ ), as polyps seldom manifest with bleeding, although, for this same reason, immunochemical FOBT has a sensitivity and specificity of $95 \%$ for detection of advanced colonic neoplasms. ${ }^{22}$

Therefore, it is necessary to shift attention earlier along the adenoma-carcinoma sequence: this task is accomplished by rectosigmoidoscopy and colonoscopy (either optical or virtual).

The rationale for rectosigmoidoscopy (also referred to as left colonoscopy if exploration is extended to the descending colon) is related to different frequency of $\mathrm{CRC}$ in the various colonic segments; in fact, CRC arises in the rectum and sigmoid colon in $55 \%$ of cases, in the descending colon in $6 \%$ of cases, in the transverse colon in $11 \%$ of cases, and in the cecum/ascending colon in $22 \%$ of cases. ${ }^{18}$ One limitation of this technique is, of course, the lack of evaluation of the entire colorectum, that may cause a considerable number of colonic adenomas and carcinomas to be missed. In recent years proximal colon $\mathrm{CRC}$ has been reported more frequently, especially in elderly individuals and females. For those reasons, whenever a colonic mass, or an advanced adenoma, or 3 or more adenomas, or a villous polyp larger than $10 \mathrm{~mm}$ is found, rectosigmoidoscopy must be followed by complete colonoscopy. ${ }^{17}$ 
OC is today regarded as the gold standard technique for CRC screening, because it allows lesion detection with both high sensitivity (90\%-100\% also for small size polyps, ie, less than $6 \mathrm{~mm}$ diameter) and high specificity. Moreover, like rectosigmoidoscopy, OC is not only useful for diagnostic purposes, but has also a therapeutic role as it enables polypectomy by means of a diathermic loop or hot bioptic pliers (hot biopsy), allowing lesion removal and biopsy. It is usually performed with light sedation or conscious sedation and therefore requires an in-patient setting. However, the main limitation of OC is patient intolerance, which can be due either to anatomical conditions (for example, presence of short colonic mesentery or a redundant colon may cause pain with progression of the OC probe), adhesions, or neoplastic and/or fibrotic narrowing/ obstruction of the bowel lumen. Other limitations of OC are the need for a cathartic preparation for optimal bowel cleansing, its high cost, and invasiveness. Moreover, some lesions of the colonic mucosa can be missed even by OC. ${ }^{23}$ Finally, OC is not completely free from potentially life-threatening complications, such as bowel perforation or bleeding.

DCBE is often used whenever conventional endoscopy fails to explore the entire colon, eg, because of patient intolerance and/or inability to reach the cecum on $\mathrm{OC}$ (which is not uncommon in cases of redundant bowel) (Figure 1). Compared with OC, DCBE has the advantage of less invasiveness, and therefore it is usually better tolerated by patients. However, the visualization of colonic lesions is heavily dependent on the radiographic projections made

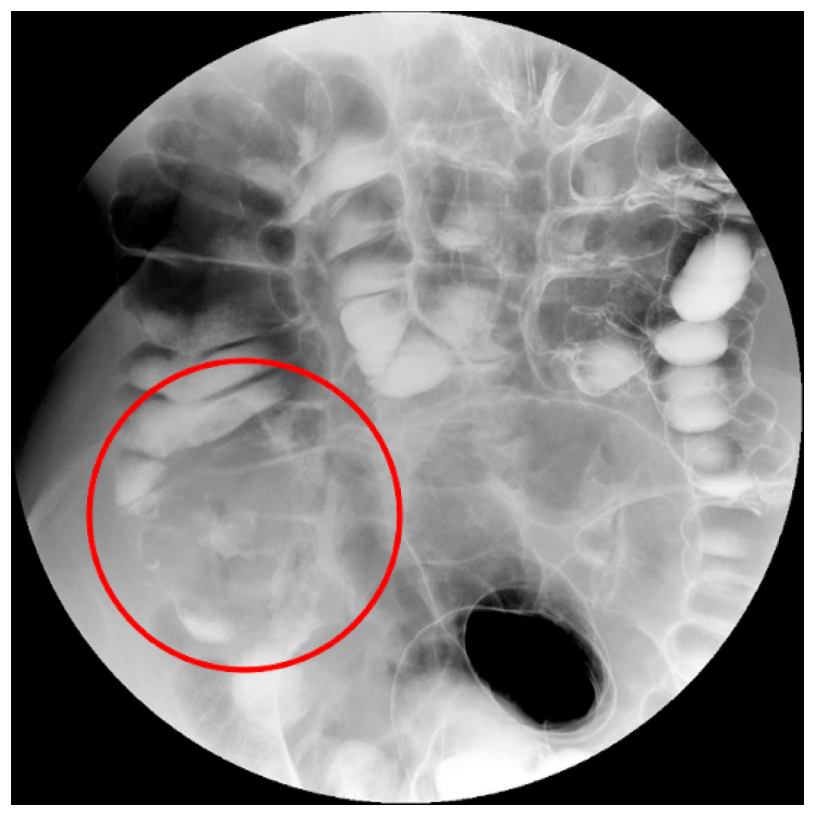

Figure I Large carcinoma of the cecum (red circle) as displayed on double contrast barium enema image. Lesion presence is inferred indirectly as a filling defect of the cecal lumen with irregular mucosal lining. to obtain DCBE images, and therefore polyps (especially smaller ones) could be masked by extracolonic structures or even by nearby colonic segments or folds, thus causing DCBE to be less sensitive than OC. Sensitivities as low as $42 \%$ for $10 \mathrm{~mm}$ polyps have been reported. ${ }^{24,25}$

Another important issue of DCBE is radiation dose, that can be quite unpredictable due to the different radiographic technique used for each patient (for example, particular anatomic conditions and/or the different time and maneuvers needed to ensure arrival of barium and air up to the cecum), but it tends to be quite high, in the order of 5 to $8 \mathrm{mSv}$. Larger patients usually require even higher radiation doses to be imaged, owing to their higher X-ray absorption. This may be a serious limitation in case of younger patients and especially for young females, who are more sensitive to ionizing radiation due to the presence of gonads inside the abdomen.

CTC has recently been included by the American Cancer Society among tests recommended for CRC screening, and has evolved from an experimental technique to a universally recognized, accurate, and validated diagnostic tool that is gaining widespread use and acceptance among both patients and referring clinicians. It is based on the acquisition of spiral CT data of the abdomen after adequate distention of the colon through rectal insufflation of gas (either air or carbon dioxide). ${ }^{26-28}$ The CT acquisition is usually performed twice: once in the supine position and then in the prone position (or vice versa): this is to optimize distention of the various colonic segments depending on gravitational compression by the surrounding abdominal structures, as well as to distinguish polyps (that are fixed to the bowel walls) from fluid and/or fecal residues (that tend to fall down due to gravitation) (Figure 2).

As CTC image formation is based on the X-ray attenuation of low-density, high-intrinsic-contrast objects (such as the air contained in the colonic lumen vs the large bowel walls, acting

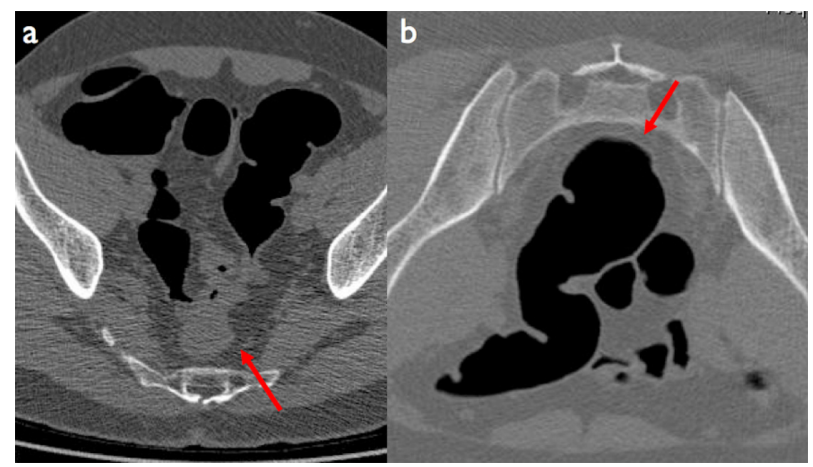

Figure 2 Computed tomography colonography image in the supine a) and prone b) position. In the supine position the collapsed sigmoid colon may mimic cancer, while on the prone position the bowel walls and the lumen are shown to be normal (red arrows). 
as an interface between intraluminal air and the extraluminal compartment), low X-ray energy is sufficient to achieve diagnostic CTC images, resulting in a low radiation dose. In other terms, if CTC is aimed at the sole examination of the colon (eg, for CRC screening purposes), use of low-radiation-dose $\mathrm{CT}$ acquisition protocols is warranted. Conversely, regulardose CT protocols will be used if CTC is part of a CT examination in which all abdominal organs are to be investigated. This is the case, for example, of CTC in patients with known $\mathrm{CRC}$ and incomplete $\mathrm{OC}$, in whom CT plays a role both for complete assessment of the colonic lumen and for oncological staging (including search of lymph node and liver metastases, or peritoneal carcinomatosis) (Figure 3).

For adequate colonic distention to be achieved, air or carbon dioxide is usually delivered into the patient's colon by means of a thin rectal catheter prior to CTC data acquisition. Insufflation can be accomplished either manually or via automatic insufflators, which allow a continuous and reliable measurement of the volume and pressure of insufflated gas. Air has the advantage of no cost and ease of administration, but is less tolerated because it is not absorbed by the colonic mucosa. Conversely, carbon dioxide is more comfortable (after completion of the exam) as it is gradually absorbed by the colonic walls, although larger volumes must be supplied compared with air. In practical terms, administration of 1 to $1.5 \mathrm{~L}$ of air or 3 to $4 \mathrm{~L}$ of carbon dioxide is usually sufficient. ${ }^{28}$

Colonic distention is also favored by parenteral administration of spasmolytic agents, such as glucagon or hyoscine-Nbutyl bromide, which inhibit peristalsis and reduce the tone of the parietal musculature. Such agents are usually administered

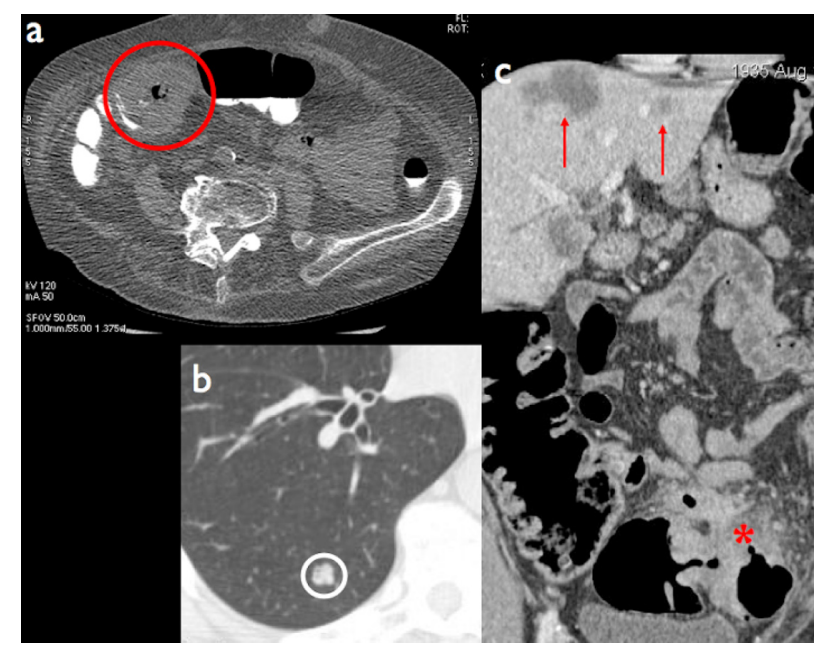

Figure 3 a) Annular stenosing cancer of the right colonic flexure (red circle) using a low dose computed tomography colonography (CTC) protocol. b, c) CTC can be performed with a regular dose protocol for detection of extracolonic disease, such as lung (b: white circle) and liver metastases (c: red arrows) in a patient with locally advanced colorectal cancer (red asterisk). intramuscularly or intravenously prior to insufflation. Contraindications to hyoscine-N-butyl bromide are glaucoma, prostatic hypertrophy, and heart rhythm disorders, whereas glucagon is contraindicated in patients with elevated blood glucose levels. In the United States (where glucagon is the only option) most experts have stopped using it.

By orally administering positive contrast material into the large bowel (barium or iodine), fecal and fluid tagging can be performed, helping to distinguish fecal/fluid residues from parietal polyps. Tagged residual fluid can then be electronically removed from CTC images by means of dedicated software. Research is in progress on subtracting solid tagged stool in patients who undergo no cathartic cleansing.

In contrast with the $2 \mathrm{D}$ plain film nature of $\mathrm{DCBE}$ images, one great advantage of CTC is the fact that cross-sectional images of the abdomen are acquired, which allows analysis of the various anatomical structures (eg, colonic lumen and content vs bowel walls, and the several components of the extracolonic compartment) without the superimposition of the surrounding organs and tissues. CTC also allows quantitative measurements of CT densities (such as that of fat for the normal ileo-cecal valve, or parenchymal density for extracolonic tissues or colonic lesions), as well as quantitative geometrical information, such as distances, areas, or volumes, without the projective distortion that is typical of radiographic images.

In order to achieve a high spatial resolution (which is essential for detection of small polyps and for high quality $2 \mathrm{D}$ and $3 \mathrm{D}$ image processing), thin slice acquisition is mandatory for CTC examinations. With current multidetector row CT equipment (64 detector rows and beyond), the entire abdomen can be scanned in less than 10 seconds with submillimeter slice thickness, resulting in voxel isotropy and elimination/ minimization of motion artifacts. ${ }^{29}$

$2 \mathrm{D}$ and $3 \mathrm{D}$ image reconstructions are necessary components of any CTC exam, as they allow assessment of large bowel anatomy on multiple planes (either orthogonal [axial, coronal, and sagittal planes], or user-defined oblique planes), as well as provision of a volumetric depiction of normal anatomy and polyp morphology. 3D reconstructions enable accurate quantification of polyp volume, which can be helpful in a follow-up to assess growth of the polyp. With modern multidetector CT equipment and powerful image processing workstations, 2D (multiplanar reformation) and $3 \mathrm{D}$ reconstructions (volume rendering) are now part of the routine diagnostic workflow of CTC. It is also possible to perform volume rendering from an intraluminal perspective, thus simulating endoscopic navigation (virtual endoscopy), or 
to 'open up' the colon and generate dissected views (virtual dissection) (Figures 4 and 5).

In the systematic review by Halligan et al in $2005,{ }^{30}$ who assessed 24 studies for a total of 4181 patients, average CTC sensitivity for large polyp ( $1 \mathrm{~cm}$ or above) detection was $93 \%$ with an average specificity of $97 \%$. For large and medium size polyps ( $6 \mathrm{~mm}$ or above), average sensitivity and specificity were both $86 \%$, while for polyps of all sizes, sensitivity ranged from $45 \%$ to $97 \%$ and specificity from $26 \%$ to $97 \%$. CTC sensitivity for detection of CRC was $96 \%{ }^{30}$

In another systematic review of the literature by Mulhall et al, ${ }^{31}$ results were substantially similar to those by Halligan et al. ${ }^{30}$ In summary, CTC has been shown to be highly sensitive for CRC detection, as well as highly specific for detection of small size polyps, which makes it suitable as a CRC screening tool. ${ }^{32,33}$

\section{CTC vs DCBE: radiation dose issues}

Few studies have compared the radiation dose of CTC and DCBE. ${ }^{34,35}$ Hirofuji et a ${ }^{34}$ measured a DCBE effective dose value of $12.7 \mathrm{mSv}$ (decreasing by $12 \%$ when digital radiography equipment was used), while an effective dose of CTC performed with a low-dose protocol was $5.7 \mathrm{mSv}$. Neri et al managed to perform successful CTC examinations for CRC screening purposes with an even lower radiation dose $(2.2 \mathrm{mSv})$ by using a combination of low exposure settings and tube current modulation. ${ }^{36}$ The lower radiation dose of CTC compared with DCBE is due to the fact that with CTC, fluoroscopy is not needed and data acquisition is performed in the supine and prone positions only. Single-acquisition protocols are currently discussed (eg, in the pediatric population), which would actually halve radiation exposure. ${ }^{37}$

According to the radiological risk figures of the International Commission on Radiological Protection, ${ }^{38}$ an average effective dose value of around $2 \mathrm{mSv}$ for CTC is associated with a theoretical $0.005 \%$ risk of lethal radiation-induced

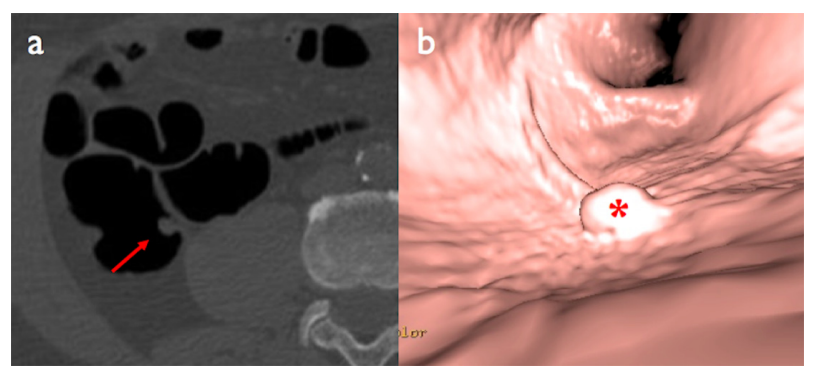

Figure 4 Sessile polyp of the ascending colon: a) native axial image (red arrow), b) virtual endoscopic view (red asterisk).

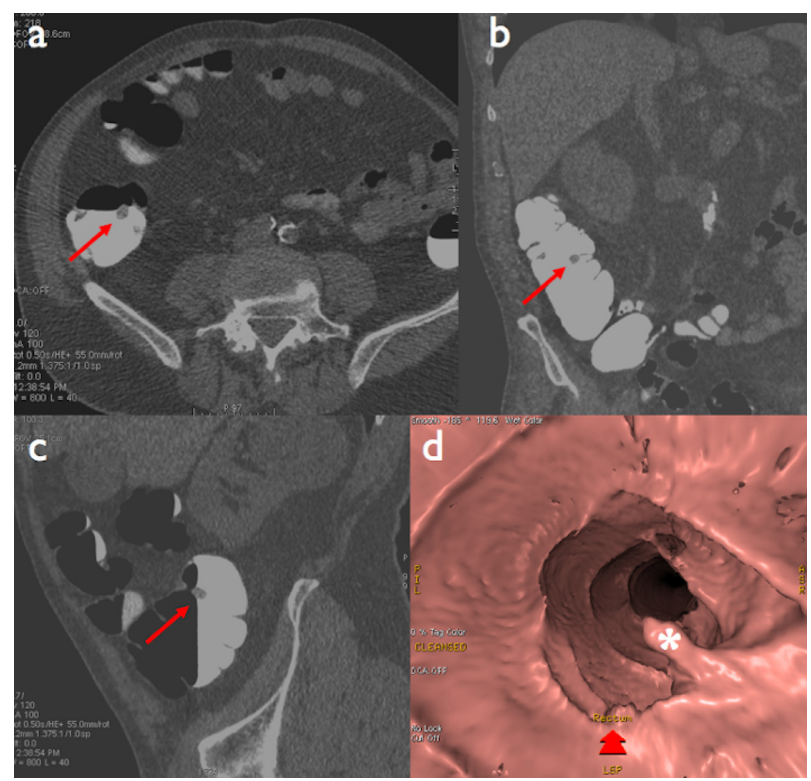

Figure 5 Pedunculated polyp of the ascending colon: a) native axial image, b) coronal reformation, c) sagittal reformation (red arrows), d) virtual endoscopic view (white asterisk).

malignancy, which further decreases with increasing patient age. Thus, CTC is an important CRC screening tool especially in middle-aged and elderly patients, in whom the potential benefit of early diagnosis of CRC far outweighs the risk associated with radiation exposure.

\section{Computer aided diagnosis}

Computer aided diagnosis (CAD) tools are software applications designed for assisting the radiologist in the diagnosis of several conditions; for detection of lung nodules on chest $\mathrm{X}$-ray or CT, breast nodules in screening mammography, and polyps in CT colonography. ${ }^{39-41}$

As CTC continues to evolve and improve, its use is shifting from highly specialized academic centers to community hospitals and nonacademic radiology practices. ${ }^{42,43}$ Thus, many radiologists are experiencing pressure from clinical colleagues to offer CTC as part of the routine services provided in their practices.

In parallel, modern multidetector CT scanners can generate data with unprecedented spatial resolution (down to $0.5 \mathrm{~mm}$ ), with a consequent enormous increase in image number; therefore, manual reading of CTC images may become error-prone due to reader fatigue. ${ }^{44}$ Moreover, image interpretation is subject to reader bias, and no systematic method has been devised so far for lesion visualization (either 2D or 3D). ${ }^{45}$

CAD systems have been developed in an attempt to overcome the limitations of software-unassisted reading of 
CTC datasets. Such applications include dedicated algorithms for automatic detection of geometrical properties of image objects (such as the shape of colonic polyps and their relationship with the bowel wall) and CT density of the normal colon and polyps. The output of those algorithms is used to create a list of 'candidate' polyps, which are shown on 2D and 3D reconstructions and native CTC images, and which must be validated or rejected by the user. CAD systems can be used in a first-reader or second-reader paradigm, depending on CAD being switched on before or after examination of CTC datasets by the human reader. Several studies have addressed the issue of evaluating the diagnostic performance of CAD systems for CTC. They are valuable for assisting radiologists in the detection of polyps, especially for detection of small lesions. However, such an increase in sensitivity is usually paralleled by a decrease of specificity, suggesting that results of CAD systems need to be adjusted to maximize overall diagnostic accuracy. ${ }^{39-41}$

The impact of CAD as second reader on experienced readers has recently been investigated in a multireader, multicase trial. ${ }^{46}$ Thus, it would be of interest to evaluate the impact of CAD systems on the diagnostic performance of readers without dedicated CTC experience, such as readers working in nonacademic centers, involved in reading a large amount of CTC generated by a screening program. In this respect, Baker et $\mathrm{al}^{47}$ have concluded that application of a CAD system for CTC is advantageous for assisting diagnostic performance of inexperienced readers, with decreased specificity being offset by a higher increase in sensitivity. However, CAD power cannot compensate for reader inexperience, as it has been shown that use of CAD software for CTC image evaluation by inexperienced readers does not significantly increase sensitivity of individual raters, thus stressing the importance of adequate training. ${ }^{48}$

\section{Conclusion}

Colorectal cancer is one of the most frequent malignancies in the Western world, for which genetic and environmental risk factors have been identified and form the basis for disease prevention. Several imaging techniques are currently available that allow detection of colorectal polyps at an early stage of development. Among those, CT colonography is one of the most promising and will likely gain increasing importance for CRC screening purposes.

\section{Disclosure}

The authors declare no conflicts of interest.

\section{References}

1. Longo DL. Approach to the patient with cancer. In: Fauci AS, Braunwald E, Kasper DL, Hauser SL, Longo DL, Jameson JL, Loscalzo J, editors. Harrison's Principles of Internal Medicine. 17th ed. New York: McGraw-Hill Professional;2008.

2. Neri E, Giusti P, Battolla L, et al. Colorectal cancer: role of CT colonography in preoperative evaluation after incomplete colonoscopy. Radiology. 2002;223:615-619.

3. Mayer RJ. Gastrointestinal tract cancer. In: Fauci AS, Braunwald E, Kasper DL, Hauser SL, Longo DL, Jameson JL, Loscalzo J, editors. Harrison's Principles of Internal Medicine. 17th ed. New York, NY: McGraw-Hill Professional;2008.

4. Jemal A, Siegel R, Ward E, Hao Y, Xu J, Thun MJ. Cancer statistics, 2009. CA Cancer J Clin. 2009;59:225-249.

5. Ferlay J, Bray F, Sankila R, Parkin DM. EUCAN: Cancer incidence, mortality and prevalence in the European Union 1997, version 4.0. IARC CancerBase No. 4. Lyon: IARCPress, 1999.

6. Moore HG. Colorectal cancer: what should patients and families be told to lower the risk of colorectal cancer? Surg Oncol Clin N Am. 2010; 19:693-710.

7. De Campos FG, Perez RO, Imperiale AR, Seid VE, Nahas SC, Cecconello I. Evaluating causes of death in familial adenomatous polyposis. J Gastrointest Surg. 2010;14:1943-1949.

8. Hadley DW, Jenkins JF, Steinberg SM, et al. Perceptions of cancer risks and predictors of colon and endometrial cancer screening in women undergoing genetic testing for Lynch syndrome. J Clin Oncol. 2008; 26:948-954.

9. Pande M, Amos CI, Osterwisch DR, et al. Genetic variation in genes for the xenobiotic-metabolizing enzymes CYP1A1, EPHX1, GSTM1, GSTT1, and GSTP1 and susceptibility to colorectal cancer in Lynch syndrome. Cancer Epidemiol Biomarkers Prev. 2008;17: 2393-2401.

10. Pande M, Amos CI, Eng C, Frazier ML. Interactions between cigarette smoking and selected polymorphisms in xenobiotic metabolizing enzymes in risk for colorectal cancer: A case-only analysis. Mol Carcinog. 2010;49;974-980.

11. Giovannucci E. An updated review of the epidemiological evidence that cigarette smoking increases risk of colorectal cancer. Cancer Epidemiol Biomarkers Prev. 2001;10:725-731.

12. Du W, Li WY, Lu R, Fang JY. Folate and fiber in the prevention of colorectal cancer: between shadows and the light. World J Gastroenterol. 2010;16:921-926.

13. Mizoue T, Tanaka K, Tsuji I, et al; Research Group for the Development and Evaluation of Cancer Prevention Strategies in Japan. Alcohol drinking and colorectal cancer risk: an evaluation based on a systematic review of epidemiological evidence among the Japanese population. Jpn J Clin Oncol. 2006;36:582-597.

14. Park JY, Dahm CC, Keogh RH, et al. Alcohol intake and risk of colorectal cancer: results from the UK Dietary Cohort Consortium. Br J Cancer. 2010;103:747-756.

15. Macari M, Bini EJ. CT colonography: where have we been and where are we going? Radiology. 2005;237:819-833.

16. American College of Radiology. ACR Appropriateness Criteria ${ }^{\circledR}$ Colorectal Cancer Screening. http://www.acr.org/ SecondaryMainMenuCategories/quality_safety/app_criteria/pdf/ ExpertPanelonGastrointestinalImaging/ColorectalScreeningCancerDoc4.aspx. Accessed Nov 15, 2010.

17. Levin B, Lieberman DA, McFarland B, et al. Screening and surveillance for the early detection of colorectal cancer and adenomatous polyps, 2008: a joint guideline from the American Cancer Society, the US Multi-Society Task Force on Colorectal Cancer, and the American College of Radiology. CA Cancer J Clin. 2008;58: $130-160$.

18. Turner JR. The gastrointestinal tract. In: Kumar V, Abbas AK, Fausto N, Aster JC, editors. Robbins and Cotran Pathologic Basis of Disease, Professional Edition, 8th ed. Philadelphia, PA: Saunders Elsevier; 2009. 
19. Stryker SJ, Wolff BG, Culp CE, Libbe SD, Ilstrup DM, MacCarty RL. Natural history of untreated colonic polyps. Gastroenterology. 1987; 93:1009-1013.

20. Hewett DG, Kahi CJ, Rex DK. Does colonoscopy work? J Natl Compr Canc Netw. 2010;8:67-76.

21. Smith RA, Cokkinides V, Brooks D, Saslow D, Brawley OW. Cancer screening in the United States, 2010: a review of current American Cancer Society guidelines and issues in cancer screening. CA Cancer J Clin. 2010;60:99-119.

22. Zhu MM, Xu XT, Nie F, Tong JL, Xiao SD, Ran ZH. Comparison of immunochemical and guaiac-based fecal occult blood test in screening and surveillance for advanced colorectal neoplasms: a meta-analysis. J Dig Dis. 2010;11:148-160.

23. Singh H, Nugent Z, Demers AA, Bernstein CN. Rate and predictors of early/missed colorectal cancers after colonoscopy in Manitoba: a population-based study. Am J Gastroenterol. 2010;105:2588-2596.

24. Winawer SJ, Stewart ET, Zauber AG, et al. A comparison of colonoscopy and double-contrast barium enema for surveillance after polypectomy. National Polyp Study Work Group. N Engl J Med. 2000; 342:1766-1772.

25. Rockey DC, Paulson E, Niedzwiecki D, et al. Analysis of air contrast barium enema, computed tomographic colonography, and colonoscopy: prospective comparison. Lancet. 2005;365:305-311.

26. Vos FM, van Gelder RE, Serlie IW, et al. Three-dimensional display modes for CT Colonography: conventional 3D virtual colonoscopy versus unfolded cube projection. Radiology. 2003;228:878-885.

27. Kim SH, Lee JM, Eun HW, et al. Two- versus three-dimensional colon evaluation with recently developed virtual dissection software for CT colonography. Radiology. 2007;244:852-864.

28. Taylor SA, Laghi A, Lefere P, Halligan S, Stoker J. European Society of Gastrointestinal and Abdominal Radiology (ESGAR): consensus statement on CT colonography. Eur Radiol. 2007;17:575-579.

29. Faggioni L, Neri E, Cerri F, Turini F, Bartolozzi C. Integrating image processing in PACS. Eur J Radiol. 2009 Jul 18. [Epub ahead of print].

30. Halligan S, Altman DG, Taylor SA, et al. CT colonography in the detection of colorectal polyps and cancer: systematic review, meta-analysis, and proposed minimum data set for study level reporting. Radiology. 2005;237:893-904.

31. Mulhall BP, Veerappan GR, Jackson JL. Meta-analysis: computed tomographic colonography. Ann Intern Med. 2005;142:635-650.

32. Pickhardt PJ, Choi JR, Hwang I, et al. Computed tomographic virtual colonoscopy to screen for colorectal neoplasia in asymptomatic adults. N Engl J Med. 2003;349:2191-2200.

33. Macari M, Bini EJ, Jacobs SL, et al. Clinical significance of missed polyps at CT Colonography. AJR Am J Roentgenol. 2004;183:127-134.

34. Hirofuji Y, Aoyama T, Koyama S, Kawaura C, Fujii K. Evaluation of patient dose for barium enemas and CT colonography in Japan. Br J Radiol. 2009:82:219-227.
35. Kemerink GJ, Borstlap ACW, Frantzen, Schultz FW, Zoetelief J, van Engelshoven JM. Patient and occupational dosimetry in double contrast barium enema examinations. Br J Radiol. 2001;74:420-428.

36. Neri E, Faggioni L, Cerri F, et al. CT colonography versus doublecontrast barium enema for screening of colorectal cancer: comparison of radiation burden. Abdom Imaging. 2010;35:596-601.

37. Sugiyama A, Ohashi Y, Gomi A, et al. Colorectal screening with single scan CT colonography in children. Pediatr Surg Int. 2007;23:987-990.

38. 1990 Recommendations of the International Commission of Radiological Protection. International Commission on Radiological Protection publication no. 60. In: Smith H editor, Annals of the ICRP 21 (no. 1-3). Oxford: Pergamon Press; 1991.

39. Yoshida H, Dachman AH. CAD techniques, challenges, and controversies in computed tomographic colonography. Abdom Imaging. 2005; 30:26-41.

40. Taylor SA, Halligan S, Burling D, et al. Computer-assisted reader software versus expert reviewers for polyp detection on CT colonography. Am J Roentgenol. 2006;186:696-702.

41. Halligan S, Altman DG, Mallett S, et al. Computed tomographic colonography: assessment of radiologist performance with and without computer-aided detection. Gastroenterology. 2006;131: 1690-1699.

42. Burling D, Halligan S, Atchley J, et al. CT colonography: interpretative performance in a non-academic environment. Clin Radiol. 2007; 62:424-429.

43. Thomas S, Atchley J, Higginson A. Audit of the introduction of CT colonography for detection of colorectal carcinoma in a non-academic environment and its implications for the national bowel cancer screening programme. Clin Radiol. 2009;64:142-147.

44. Slater A, Taylor SA, Tam E, et al. Reader error during CT colonography: causes and implications for training. Eur Radiol. 2006;16:2275-2283.

45. Pickhardt PJ, Lee AD, Taylor AJ, et al. Primary $2 \mathrm{D}$ versus primary $3 \mathrm{D}$ polyp detection at screening CT colonography. AJR Am J Roentgenol. 2007;189:1451-1456.

46. Dachman AH, Obuchowski NA, Hoffmeister JW. Effect of computeraided detection for CT colonography in a multireader, multicase trial. Radiology. 2010;256:827-835.

47. Baker ME, Bogoni L, Obuchowski NA, et al. Computer-aided detection of colorectal polyps: can it improve sensitivity of less-experienced readers? Preliminary findings. Radiology. 2007;245:140-149.

48. Neri E, Faggioni L, Regge D, et al. CT colonography: role of a second reader CAD paradigm in the initial training of radiologists. Eur J Radiol. 2010 Sep 8. [Epub ahead of print].
Cancer Management and Research

\section{Publish your work in this journal}

Cancer Management and Research is an international, peer-reviewed open access journal focusing on cancer research and the optimal use of preventative and integrated treatment interventions to achieve improved outcomes, enhanced survival and quality of life for the cancer patient. The journal welcomes original research, clinical \& epidemiological

\section{Dovepress}

studies, reviews \& evaluations, guidelines, expert opinion \& commentary, case reports \& extended reports. The manuscript management system is completely online and includes a very quick and fair peerreview system, which is all easy to use. Visit http://www.dovepress.com/ testimonials.php to read real quotes from published authors. 